\title{
[Review] Smoking and COVID-19: Rapid evidence review for the Royal College of Physicians, London (UK)
}

\author{
David Simons ${ }^{1}$, Jamie Brown², Lion Shahab², Olga Perski \\ 1 Royal Veterinary College, RVC \\ 2 University College London, University of London
}

\section{Abstract}

SARS-CoV-2 is the causative agent of COVID-19, an emergent zoonotic disease which has reached pandemic levels and is designated a public health emergency of international concern by the World Health Organisation. Early data suggest that older age, male sex and a diagnosis of hypertension or diabetes independently increase the risk of hospitalisation and death from COVID-19; however, the biological mechanisms underpinning these associations are currently unclear. The SARS-CoV-2 virus enters human cells through the ACE2 receptor. Current and past tobacco smoking is associated with changes in the ACE2 receptor expression, hypertension, diabetes and worse outcomes following other viral infections. It is hence plausible that smoking is an independent risk factor for hospitalisation and death from COVID-19. Here, we review available evidence for an association between smoking status and hospitalisation for COVID-19. We highlight concerns regarding the quality of available data and call for more systematic collection of smoking status in future studies.

Large age and gender differences in case severity and mortality have been observed in the ongoingCOVID-19 pandemic $^{[1]}$; these differences are currently unexplained. Early evidence suggests that hypertension and diabetes are key risk factors for heightened morbidity and mortality from COVID-19.

SARS-CoV-2 has been shown to enter epithelial cells through the ACE2 recepto[2]. Some evidence suggests that gene expression and subsequent receptor levels are elevated in the airway and oral epithelium of current smokers ${ }^{[3][4][5]}$, thus putting smokers at higher risk of contracting SARS-CoV-2. Other studies, however, show that nicotine downregulates the ACE2 receptor ${ }^{[6]}$. These uncertainties notwithstanding, both past and current smoking is known to increase the risk of respiratory viral ${ }^{[7][8]}$ and bacterial ${ }^{[9][10]}$ infections and is associated with worse outcomes once infected. Cigarette smoke reduces the respiratory immune defence through peri-bronchiolar inflammation and fibrosis, impaired mucociliary clearance and disruption of the respiratory epithelium ${ }^{[11]}$. There is also reason to believe that behavioural factors (e.g. regular hand-to-mouth movements) involved in smoking may increase SARS-CoV-2 transmissions as has been observed for other viruses ${ }^{[12]}$. However, early data from the COVID-19 pandemic have not provided clear evidence for a negative impact of smoking on COVID-19 related outcomes, such as hospitalisation or mortality[13].

Available data from China suggest a lower than expected number of hospital admissions among the smoking population. Crude (non age-stratified) smoking prevalence in China is reported at 54\% among men and 2.6\% among women ${ }^{[14]}$; however, a review of 8 Chinese studies with data on smoking status suggest fewer hospitalised smokers than would be 
expected from national prevalence estimates ${ }^{[15]}$. The largest of these studies presented smoking status for 1085 patients hospitalised with COVID-19 ${ }^{[1]} .12 .6 \%$ were current smokers, $1.9 \%$ past smokers and $85.4 \%$ were never smokers. Current and past smokers had worse outcomes (defined as ITU admission or death) than never smokers at $12 \%, 24 \%$ and $4.8 \%$, respectively. Two further studies presented smoking status for 191 and 140 hospitalised patients, respectively. In the first study ${ }^{[16]}, 11$ individuals $(5.8 \%)$ were identified as current smokers with 6 surviving until discharge. The second study reported 2 current (1.4\%) and 7 past (5\%) smokers with severe disease ${ }^{[17]}$. Five further studies ${ }^{[18][19][20][21][22]}$ identified 46 current or past smokers in a combined population of 386 hospitalised patients. Across these studies, data were presented for 1,690 admitted patients, 56 of whom (3.3\%) were identified as being current or past smokers.

These early studies are limited by a number of factors. First, they describe populations admitted to intensive care in the context of an emerging epidemic; hence, it is likely that data collection on smoking status was not considered a priority. As a consequence, current smoking rates are likely to be underreported and past smoking may be conflated with never smoking. For example, past smoking was only reported in $3 / 8$ studies. In China, approximately $69 \%$ of men aged $60+$ years have a history of or are current smokers, which suggests that a non-trivial proportion of past smokers for whom a period of regular smoking earlier in life might have caused lasting disruption to the respiratory system are not taken into account. Second, individuals with severe symptoms from COVID-19 may have stopped smoking prior to admission to a care facility and may therefore not have been recorded as current smokers (i.e. reverse causality) ${ }^{[23]}$. Third, smokers with COVID-19 may be less likely to present to hospital because of lack of access to healthcare and more likely to die in the community from sudden complications (i.e. self-selection) ${ }^{[23]}$. Fourth, if we take at face value the observation that smoking appears to be substantially protective against hospitalisation for COVID-19, we would expect a female rather than male preponderance of patients in the cited studies ${ }^{[24]}$. Fifth, it should also be noted that smoking is a risk factor for both hypertension and diabetes, two diseases associated with worse outcomes from COVID-19, which suggests that current and past smoking may be both directly and indirectly implicated in COVID-19 outcomes.

In the UK, smoking status is systematically recorded in individuals admitted to hospital with respiratory illnesses. We are currently in the process of developing a case-control study based at a London acute care trust to understand the added risk of hospitalisation due to COVID-19, compared with admission for seasonal influenza or lower respiratory tract infections a year previous. We also plan to add relevant questions to a monthly population survey of the general population of adults in England, and will provide up to date statistics on www.smokinginengland.info. In the meantime, we believe that high quality smoking cessation advice should form part of public health efforts during COVID-19 (e.g. https://www.todayistheday.co.uk/. At the very least, smoking cessation reduces acute risks from cardiovascular disease and could reduce demands on the healthcare system ${ }^{[25]}$. GPs and other healthcare providers can play a crucial role brief, high-quality and free online training is available at https://www.ncsct.co.uk/vba.

\section{References}

1. ${ }^{a, b}$ Wei-jie Guan, Zheng-yi Ni, Yu Hu, Wen-hua Liang, Chun-quan Ou, Jian-xing He. (2020). Clinical Characteristics of Coronavirus Disease 2019 in China. N Engl J Med. doi:10.1056/nejmoa2002032. 
2. `Markus Hoffmann, Hannah Kleine-Weber, Simon Schroeder, Nadine Krüger, Tanja Herrler, Sandra Erichsen. (2020). SARS-CoV-2 Cell Entry Depends on ACE2 and TMPRSS2 and Is Blocked by a Clinically Proven Protease Inhibitor. Cell. doi:10.1016/j.cell.2020.02.052.

3. ^Samuel James Brake, Kathryn Barnsley, Wenying Lu, Kielan Darcy McAlinden, Mathew Suji Eapen, Sukhwinder Singh Sohal. (2020). Smoking Upregulates Angiotensin-Converting Enzyme-2 Receptor: A Potential Adhesion Site for Novel Coronavirus SARS-CoV-2 (Covid-19). JCM, vol. 9 (3), 841. doi:10.3390/jcm9030841.

4. ` Guoshuai Cai. (2020). Bulk and Single-Cell Transcriptomics Identify Tobacco-Use Disparity in Lung Gene Expression of ACE2, the Receptor of 2019-nCov. doi:10.20944/preprints202002.0051.v3.

5. ^ Joan C Smith, Jason Meyer Sheltzer. (2020). Cigarette smoke triggers the expansion of a subpopulation of respiratory epithelial cells that express the SARS-CoV-2 receptor ACE2. doi:10.1101/2020.03.28.013672.

6. ^ Joshua M. Oakes, Robert M. Fuchs, Jason D. Gardner, Eric Lazartigues, Xinping Yue. (2018). Nicotine and the reninangiotensin system. American Journal of Physiology-Regulatory, Integrative and Comparative Physiology, vol. 315 (5), R895-R906. doi:10.1152/ajpregu.00099.2018.

7. ^ Justin T Denholm, Claire L Gordon, Paul D Johnson, Saliya S Hewagama, Rhonda L Stuart, Craig Aboltins. (2010). Hospitalised adult patients with pandemic (H1N1) 2009 influenza in Melbourne. Australia. Medical Journal of Australia, vol. 192 (2), 84-86. doi:10.5694/j.1326-5377.2010.tb03424.x.

8. `Tochukwu Raphael Abadom, Adrian D. Smith, Stefano Tempia, Shabir A. Madhi, Cheryl Cohen, Adam L. Cohen. (2016). Risk factors associated with hospitalisation for influenza-associated severe acute respiratory illness in South Africa: A case-population study. Vaccine, vol. 34 (46), 5649-5655. doi:10.1016/j.vaccine.2016.09.011.

9. ^ Jordi Almirall, Carlos A. González, Xavier Balanzó, Ignasi Bolíbar. (1999). Proportion of Community-Acquired Pneumonia Cases Attributable to Tobacco Smoking. Chest, vol. 116 (2), 375-379. doi:10.1378/chest.116.2.375.

10. ^ Charles Feldman, Ronald Anderson. (2013). Cigarette smoking and mechanisms of susceptibility to infections of the respiratory tract and other organ systems. Journal of Infection, vol. 67 (3), 169-184. doi:10.1016/j.jinf.2013.05.004.

11. ^ J A Dye, K B Adler. (1994). Effects of cigarette smoke on epithelial cells of the respiratory tract..Thorax, vol. 49 (8), 825-834. doi:10.1136/thx.49.8.825.

12. ^ Paloma I. Beamer, Kevin R. Plotkin, Charles P. Gerba, Laura Y. Sifuentes, David W. Koenig, Kelly A. Reynolds. (2015). Modeling of Human Viruses on Hands and Risk of Infection in an Office Workplace Using Micro-Activity Data. Journal of Occupational and Environmental Hygiene, vol. 12 (4), 266-275. doi:10.1080/15459624.2014.974808.

13. 'Constantine Vardavas, Katerina Nikitara. (2020). COVID-19 and smoking: A systematic review of the evidence. Tob. Induc. Dis., vol. 18 (March). doi:10.18332/tid/119324.

14. `Shiwei Liu, Mei Zhang, Ling Yang, Yichong Li, Limin Wang, Zhengjing Huang. (2016). Prevalence and patterns of tobacco smoking among Chinese adult men and women: findings of the 2010 national smoking survey. $J$ Epidemiol Community Health, vol. 71 (2), 154-161. doi:10.1136/jech-2016-207805.

15. ^ Konstantinos Farsalinos, Anastasia Barbouni, Raymond Niaura. (2020). Smoking, vaping and hospitalization for COVID-19. Qeios. doi:10.32388/Z6908A.10.

16. ^ Fei Zhou, Ting Yu, Ronghui Du, Guohui Fan, Ying Liu, Zhibo Liu. (2020). Clinical course and risk factors for mortality of adult inpatients with COVID-19 in Wuhan, China: a retrospective cohort study. The Lancet, vol. 395 (10229), 1054- 
1062. doi:10.1016/s0140-6736(20)30566-3.

17. ^ Jin-jin Zhang, Xiang Dong, Yi-yuan Cao, Ya-dong Yuan, Yi-bin Yang, You-qin Yan. (2020). Clinical characteristics of 140 patients infected with SARS-CoV-2 in Wuhan, China. Allergy. doi:10.1111/all.14238.

18. `Wei Liu, Zhao-Wu Tao, Wang Lei, Yuan Ming-Li, Liu Kui, Zhou Ling. (2020). Analysis of factors associated with disease outcomes in hospitalized patients with 2019 novel coronavirus disease. Chinese Medical Journal. doi:10.1097/cm9.0000000000000775.

19. ^ Chaolin Huang, Yeming Wang, Xingwang Li, Lili Ren, Jianping Zhao, Yi Hu. (2020). Clinical features of patients infected with 2019 novel coronavirus in Wuhan, China. The Lancet, vol. 395 (10223), 497-506. doi:10.1016/s0140$6736(20) 30183-5$.

20. ^Pingzheng Mo, Yuanyuan Xing, Yu Xiao, Liping Deng, Qiu Zhao, Hongling Wang. (2020). Clinical characteristics of refractory COVID-19 pneumonia in Wuhan, China. doi:10.1093/cid/ciaa270.

21. `Wanbo Zhu, Kai Xie, Hui Lu, Lei Xu, Shusheng Zhou, Shiyuan Fang. (2020). Initial clinical features of suspected Coronavirus Disease 2019 in two emergency departments outside of Hubei, China. J Med Virol. doi:10.1002/jmv.25763.

22. ^ Jiong Wu, Xiaojia Wu, Wenbing Zeng, Dajing Guo, Zheng Fang, Linli Chen. (2020). Chest CT Findings in Patients with Corona Virus Disease 2019 and its Relationship with Clinical Features. Investigative Radiology. doi:10.1097/rli.0000000000000670.

23. a, b Lion Shahab, Robert West, Jamie Brown. (2020). Review of "Smoking, vaping and hospitalization for COVID-19". Qeios. doi:10.32388/N5BBEF.

24. ^ John Stapleton. (2020). Review of "Smoking, vaping and hospitalization for COVID-19". Qeios. doi:10.32388/Z79ET6.

25. ^ Lindsay F Stead, Diana Buitrago, Nataly Preciado, Guillermo Sanchez, Jamie Hartmann-Boyce, Tim Lancaster. (2013). Physician advice for smoking cessation. doi:10.1002/14651858.cd000165.pub4. 